Irada Y. Alakbarova

DOI: 10.25045/jpis.v08.i2.05

Institute of Information Technology of ANAS, Baku, Azerbaijan

airada.09@gmail.com

\title{
IMPACT OF THE WIKI CONCEPT ON THE FORMATION OF THE KNOWLEDGE SOCIETY
}

The article describes the basic principles of the wiki concept and identifies the similarities between the knowledge society and wiki-society. It also explores the hierarchy of Wikipedia management and control and analyzes the solution of some problems. The results of the analysis set forth the role of the wiki-concept in tackling problems identified in this study.

Keywords: wiki technologies, wiki-society, information society, knowledge society, collective knowledge.

\section{Introduction}

The development prospects of the modern society are closely linked to the development of information and communication technologies (ICT), information processing and knowledge production. The development of ICT provides new opportunities for high speed data transmission, data access, efficient use of various information sources, and processing of large volumes of data. Today, one of the main tendencies of the frameworks, implemented by the countries for the development of the information society, is the provision of modern ICT tools for the population, and enhancing their skill to use it efficiently. The development of the Internet has transformed it to a standard channel for social communications. Different trade transactions, cash flows and other information exchanges are carried out across the global network. New social networks, virtual groups and societies are emerging; new psychological image of people are forming. The knowledge society, electronic society, network society, and other modern scientific trends are studied in a broad spectrum in order to determine the features, problems and perspectives of these social processes.

The capabilities of the Internet have generated a huge amount of information resources such as social media. Social media is an opportunity to establish a virtual social communication and share information with others. Social media is a tool that affects the development of knowledge society, social-cultural relations, economic relations, technology and education. Modern technologies, such as Big Data, cloud computing, and wiki, have a profound impact on knowledge, its generation, storage and processing techniques. The Knowledge Society is gaining new opportunities with the development of social media. These opportunities, mainly, cover the process of generating collective knowledge. Bringing collective knowledge to science leads to the formation of a knowledge society and the development of the information economy, which in turn, increases the demand for information resources. In this process, the intelligent human capital and qualified specialists play a significant role.

Wiki technology, which is one of the key areas of social media, is of particular importance. Wiki technologies aim at giving every Internet user the opportunity to create a web page at any time, modify existing pages and download files of various formats. Wiki technology allows users to create content on the Internet as a full-fledged web-programmer, regardless of their specialty and interests, to participate in discussions, to express their attitude on the content created and modified by other users. This, in turn, leads to the emergence of new social relationships in the society. Wiki technology has also led to the emergence of a new type collaboration of the Internet users, i.e., the Wiki Community [1]. Wiki-community allows the people to socialize, generate collective knowledge, and share this knowledge. The main purpose of this study is to identify the specific features of the knowledge society and justify its compatibility with the wiki concept, and to determine the role of wiki technologies in the formation of a knowledge society. 


\section{Wiki concept}

The Wikipedia virtual encyclopedia based on wiki-technologies was established in 2001. Today, it is one of the most popular Internet projects in the world. It serves as a new model in creating, publicizing, expanding and maintaining the relationships between the citizens and knowledge holders [2]. Achievements of Wikipedia and its projects, which is one of largest information sources of the world, prove that the modern Internet users do not want to be passive users of the virtual environment. They intend to freely create web pages, dynamically change the information on existing pages, work with files of different formats and share with others [3].

The wiki concept, developed by the American web-developer Ward Cunningham in 1995, has promoted the new opinions to the content accumulated in virtual space, and revolutionized the social relationships and formation and distribution of knowledge. The Wiki concept is one of the aspects of Web 2.0 technologies and characterizes the infrastructure that reflects the collaboration of users, network coordination and the social aspects of real life. Web 2.0 technologies cover the following fields [4]:

- Information management: search, evaluation, and storage;

- Behavior of Internet users in the virtual space;

- Relationship management: establishing relationships in virtual space, establishing and supporting virtual communities, sharing information.

The growing wiki-projects are distinguished by the fact that they are more transparent and accessible than the traditional Web 2.0 technologies. It should be taken into account that, in the social media tools, such as forums, social networks, blogs, and micro blogs, the author can hide some problems from the readers by deleting the uploaded text or file. The deleted or hidden information in the Wiki projects can be easily detected and recovered on the server's database by any Internet user. Thus, the project database is open to everyone. All discussions are open and everyone can take full advantage of the opportunities provided by Wiki technologies. In contrast to other traditional social networks, the wiki-based projects are considered to be the most successful in organizing collective knowledge bases $[5,6]$.

The Wiki technology is based on the hyperlinks. When a user creates a new content or website, the content will be enriched by other users after a certain period of time. The topic is linked to various related web pages the through various hyperlinks. Over time, the web content enriched with different content becomes ultimately an information resource with certain knowledge. In most cases, this recalls the process of repetition and revising. Collectively generated knowledge is beneficial both for the creators of this knowledge and the readers. Ward Cunningham and Bo Leuf sheds the light on the essence of the wiki concept in their book The Wiki Way: Quick Collaboration on the Web [7,8]:

- Wiki invites all the Internet users to participate in the generation of virtual knowledge using only a browser.

- Wiki provides the links between the web pages covering a variety of topics. It is not important whether these pages exist or not. The references to non-existing wiki-pages will soon lead to their development.

- Wiki prefers collective knowledge in the creation and modification of an encyclopedic article. It is anticipated that collectively generated knowledge is more complete and objective.

The Wiki concept has also stimulated the development of new views such as wikinomics, wiki-democracy, and wiki-parliament, which cover different spheres of society. Wikinomics is a new attitude supporting the public relations and mutual opinions in economic approaches and management. An expert in information technology well-known Canadian scientist Donald Topscott states, in the book Wikinomics: How Mass Collaboration Changes Everything, the business of the future will be characterized in two aspects: the closeness will be substituted by 
transparent relationship and companies will try to attract foreign experts to deal with the complex production issues [9].

Scientists are still pondering why the Wiki environment attracts the knowledge carriers of the society and why the people are willing to donate their labor, knowledge and time to the world community through wiki-projects for free $[5,9,10]$. The main reason for this is the increasing role of the content created by the wiki-community in education, social and political issues and its impact on the public opinion. Wiki-environment is a place, in which the users collectively work and want to convey their attitude on the processes taking place in society, their ideological outlook, and the knowledge they hold. It is a medium, through which knowledge and information is produced, processed, disseminated and utilized the wiki technologies. Studies show that the topics of the information gathered in Wiki environment reflects the knowledge and outlook of the users [10-12]. Wiki-community members prefer not to include the information required in the wikipages, but the information they are interested in or they know better. This feature affects the volume, relevance, and quality of information on the wiki pages, and creates a sharp distinction between the wiki-pages in terms of information capacity and quality.

The development of wiki-projects depends on the collective activity of the users. Various ways are used to involve the users in the collective knowledge-building process, most of which gives full freedom to the user for content building.

\section{Knowledge and Knowledge Society}

The concept of "knowledge society" was first used by the American politician, professor of Yale University Robert Lane in 1966 [12]. Although the concept was widely studied by the American economist, sociologist Peter Drucker in 1969, [13] it was used in scientific literature only in the 90 s of the twentieth century.

The concept of "knowledge society" or "knowledge-based society" has certain characteristics that differ from the information society. These characteristics include the realization methods of knowledge, its role in socio-cultural relationships and being a subject of manipulation on global security. In this regard, the international relations between the states and public organizations are strengthened. Furthermore, there is a need for the development and implementation of new schemes of interaction between the parties interested in a successful and sustainable transition into the information society [14].

P.Drucker explains the knowledge society by the following essential conditions $[13,15]$ :

1. Knowledge producers must work as a team.

2. Knowledge producers must have the experience of freely learning and acquiring knowledge.

3. If knowledge-makers are not members of the same organization, they should act as members of an organization.

P.Drucker mentions that the knowledge-maker needs to be governed as a physical laborer and has to have intellectual abilities. These skills include engineering, mathematics, manufacturing and technology skills. Moreover, social skills - the organization of work in the collective and the creative peculiarities are also distinguishing features of the knowledge worker. Thus, intellectual abilities of the knowledge workman should prioritize. The author gives a clear idea of advanced training and uninterrupted education by introducing the concept of "educated society" in his work [15].

Oxford University professor M.Earl specifies various forms of knowledge:

- science: laws, theories and procedures adopted by everyone;

- opinion: political rules, probability settings and heuristics;

- practice: information obtained from activities and observations used in scientific research.

M. Earl emphasizes the knowledge hierarchy in the knowledge analysis and states that experience can be regarded as potential knowledge, and it is based on memory, while the opinion 
is knowledge at the stage of realization and based on the analysis and understanding. When it comes to the science, it is the knowledge recognized by all and based on correct formulation and consensus [16].

The UNESCO report "Toward Knowledge Societies" published in 2005 states that, today, knowledge has become the subject of major economic, political and cultural interests and can be a quality indicator of the society. The report, prepared by world-renowned experts in economics, sociology and philosophy, Dr. M. Castells, J. Derrida, P. Riker and A.Turen, states: "Social isolation in societies is unacceptable, since knowledge is a public property which is open to everyone" [17]. Here, knowledge is defined as a human ability to effectively use information. In general, knowledge is information about a studied and learned object, i.e., a special and ideal form of processed information as a result of certain activities. Knowledge Society is a knowledge-based society, i.e., a part of society produces, collects and processes knowledge, and another part applies it to practice in different ways.

The report states that the concept of information society depends on the development of technology, while the knowledge society is broader - social, ethical, cultural and political. In the report, the lifelong learning of human beings is related to the concept of "learning society", and the current situation requires the human to learn all his/her life. This process is performed on three levels: a) personal and cultural, b) occupational, and c) social and civil [17].

\section{Similar and distinctive features of wiki community and knowledge society}

From the aforementioned, it can be concluded that the knowledge society requires people to work in teams and learn lifelong. These features are the basic principles of the wiki concept. Thus, the wiki-community requires collective activities of the social groups consisting of the people with different demographics ideological views and activities. The key principles of Wikipedia, as M. Earl states, are properly expressing the information, basing on the consensus, and the fact that knowledge is open to everyone [18]. Hence, it can be used to develop Internet projects for wikicollective knowledge-building. Wiki is also a tool that brings together knowledge carriers and enables the social networking of knowledge communities. In the knowledge society the knowledge-makers are knowledge workers, while in wiki community, collective knowledge is build by the wiki-users. However, the main activity of both societies, regardless of their name, is the same. This means that wiki-users are also knowledge-makers. They constantly learn, collect information together and share their knowledge with others. As a result, as stated in the report "Toward Knowledge Societies", a new global information culture is emerged, which eradicates the borders between the knowledge producers and users. Wiki-community has been organized in such a way that all processes are managed collectively, and a balance has been established between the intellectual property and public domain spread.

Table 1, the comparison of similar and distinctive features of the knowledge society with the wikicommunity shows that they are very similar. The opportunities for free and collective knowledge building and its dissemination have transformed the wiki environment into a giant knowledge base.

One of the main features of the knowledge society is its mobility. Thus, geographical location, social status, nationality, and other personal features of knowledge-makers do not play a significant role in the knowledge society. The wiki-community acting in the wiki projects also enclose these features. The factors that unite the people in the wiki-society are their interests, knowledge and ideological views. Though the geographical location is insignificant in such a society, the language factor is important for the communication. It should be taken into account that, today, Wikipedia provides articles in 295 languages, and anyone who wants to convey the knowledge to the world community should incorporate the information in several leading languages of the world. Each language segment of Wikipedia has wiki-societies. Although, these wiki-societies are not dependent on the wiki-societies in other language sections, they also share experiences and work together to solve various problems. 
Table 1.

Similar and distinctive features of the wiki society and the knowledge society

\begin{tabular}{|l|l|l|}
\hline Features & Knowledge Society & Wiki-society \\
\hline Participants & Knowledge-generators & Wiki-users \\
\hline Knowledge acquisition form & Free & Free \\
\hline Knowledge-building form & Collective & Collective \\
\hline Social-demographic situation & Mobile & Mobile \\
\hline Language Factor & Optional & Required \\
\hline Information & Required & $\begin{array}{l}\text { Depending on the Areas of } \\
\text { Interest }\end{array}$ \\
\hline Generated knowledge quality & High & High ,medium and poor \\
\hline $\begin{array}{l}\text { Relation with state or public } \\
\text { body }\end{array}$ & $\begin{array}{l}\text { May belong to any state or } \\
\text { public body }\end{array}$ & $\begin{array}{l}\text { Do not belong to any state or } \\
\text { public body }\end{array}$ \\
\hline
\end{tabular}

On the other hand, decisions in science and technology traditionally depend on the relationships between the scientists and their organizational structures, the government and the entrepreneurs. The society is obliged to agree with any decision that has been made. However, in the knowledge society, decision-making is carried out with the consent of scientists, research institutions, decision makers, citizens and societies in the general consensus and understanding, which also reflects the wiki principles.

\section{Management and control of Wikipedia project}

The Wiki-community often means Wikipedia and the Internet users participating in its projects. But it should be taken into consideration that there are hundreds of projects controlled by wiki technologies in the virtual space (Wikitravel, LyricWiki, Wikireality, Wikisource, Encyclopedia of Life, Socionics, CFD Online etc.) and their quantity is constantly growing. Each wiki-project has wiki-communities, which collectively operate in accordance with their own rules.

In the wiki-communities, which have the largest coverage in the world, people are discussing all the issues, from the problems of the country in which they live, to the activities of government agencies and the latest developments in the scientific environment. This community is dynamic, and has created a new social environment by attracting millions of users. Therefore, today, it is a profitable, highly effective, extensive and ever-growing society. Any community, which skillfully take advantage of advanced ICT tools and deeply understands the philosophy of Wikipedia and unites in a wiki-community, is free from commitments, i.e., it can not be threaten by any administrative action or subordinated to any government agency.

Wikipedia and its projects are run by the Wikimedia Foundation, which is a nongovernmental organization [19]. The Wikimedia Foundation manages the activity of a wikicommunity consisting of the active Wikipedia users. Since Wikipedia is based on the volunteer activity, the self-governance principle plays an important role here. Although everyone within the wiki-environment has the ability to operate actively, the wiki-community members are categorized by their activities: top designers, stewards, bureaucrats, administrators, registered users, bots, and anonymous members $[19,20]$. Those who use Wikipedia only for information are not included in the Wikimedia community and called passive users. These users are not registered and do not make any changes to the articles.

The top project designers of Wikipedia are entitled to control all its projects and, if necessary, have the right to change the structure and even the system of the site. Some of the top designers have the right to access the Wikipedia servers. Stewards are global administrators, i.e., regardless 
of the language, they control all Wikipedia projects. Bureaucrats are the users who have the technical capabilities to provide the rights of administrators and bureaucrats to the other users. Administrators have greater technical capabilities than the ordinary users. They control wiki-pages and can restrict the user activity that violates the Wikipedia rules. Administrators also have the authority to modify the general interface of Wikipedia and manage the system information [20].

Registered users have more rights than the anonymous users. For example, they can set up the site's interface and participate in the discussions with personal nicknames and create personal pages. The unregistered users are called "anonymous". Since the Internet Protocol (IP) addresses of the computer used by the anonymous computers are stored in the system, the users can easily determine the country in which the anonymous users operate. Anonymous users can also create new articles as the regular users and make changes to existing articles. However, anonymous users do not have the right to upload pictures, videos and other files to wiki pages, as opposed to registered users.

The bots are the pseudo users, i.e., automated programs, performing the technical work, such as editing spelling mistakes in articles, organizing the internal links between the wiki pages, controlling the proper layout of templates, and deleting spam. The bot is a shortened form of the word "robot". They are created by the Wikipedia users and intended for faster editing. Any user can have several bots. The bots are created in Python, PHP, Java and Perl programming languages. It is not required to be a programmer to create a bot. Special Wikipedia pages provide guides and examples to create a bot $[20,21]$.

The registered Wikipedia users often re-register with different names. Such registration names are called "sock puppetry". Although Wikipedia permits to create "puppets", it is not welcomed. Those who have a puppet should inform others about it by writing on their personal pages. It is forbidden to use the puppets in the voting and debate of Wiki-community. Taking into consideration all abovementioned, the hierarchical structure of the wiki-community can be given as in Figure 1.

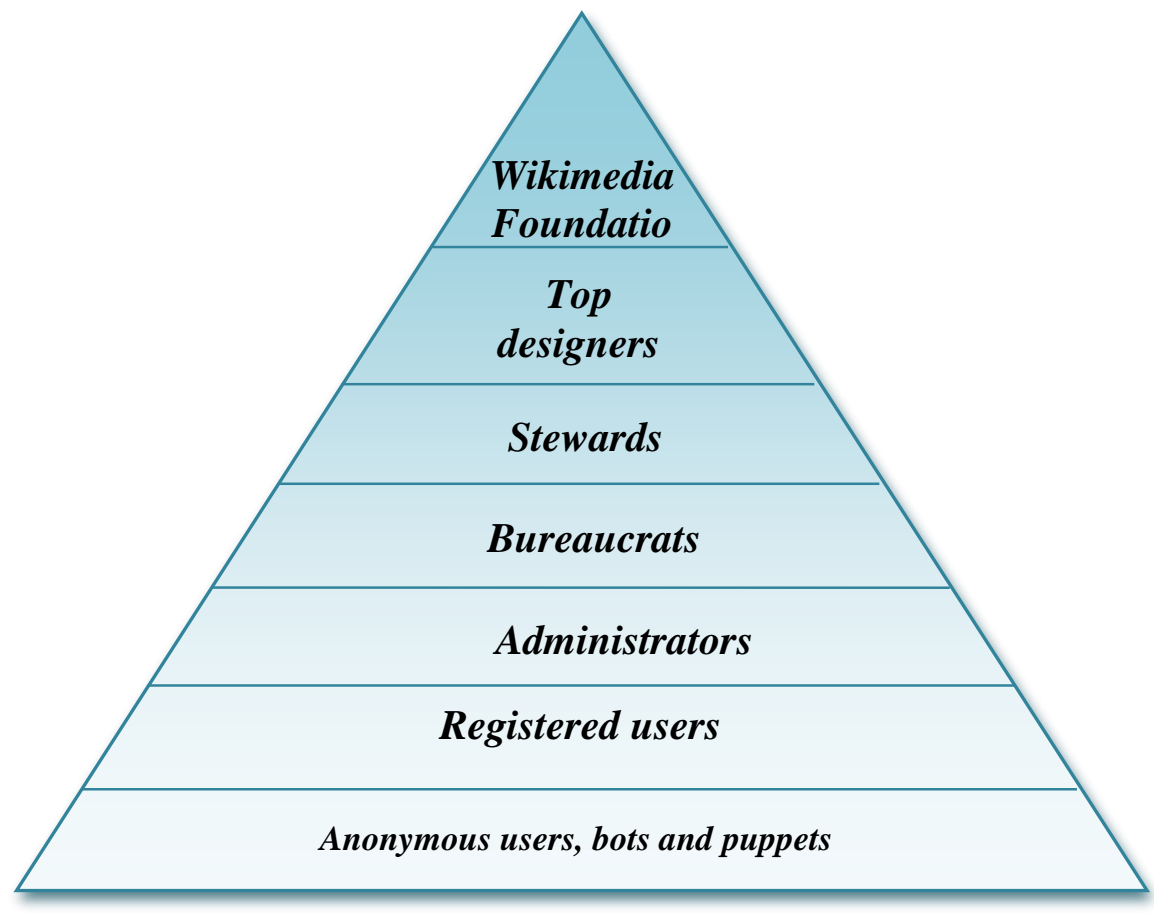

Figure 1. Hierarchy of management and control of Wikipedia

The hierarchical structure of the wiki-community provides a general idea of those who make up this society. However, unless the technical capabilities of the wiki users are considered, the following categories of the wiki members can be distinguished: 
1. Professionals and scientists who want to voluntarily convey their knowledge to people;

2. Those who want to acquire additional knowledge and have a direct contact with the knowledge carriers;

3. Those who want to create new social relationships and wiki-projects by taking advantage of vigorous opportunities provided by the wiki environment;

4. Those who use wiki-technologies to disseminate any political and ideological view.

The hierarchical structure of the Wikimedia community clarifies the essence, features, and principles of Wikipedia. The Wikimedia users aim to identify the key factors affecting the formation of a knowledge society in the wiki environment.

\section{Perspectives of using the wiki experience in the knowledge society}

Previous studies unveiled that wiki-community is a society that provides the mass production of information and knowledge, and provides its dissemination. Moreover, it regulates and "filters" information and knowledge. The key trends in the knowledge society and the role of wiki concepts in solving the issues are as follows:

1. Knowledge Society is composed of the people who are capable to freely learn. ICT assist them to develop their professional skills. Thus, these technologies accelerate the process of creating and disseminating the knowledge. One of the key issues in the knowledge society is the right behavior in the information flow, i.e., the ability to quickly and easily acquire, process, transmit the information and to select the useful information out of the useless or harmful information. These issues are also relevant in wiki projects. For example, in Wikipedia, people should have the ability to freely learn, gain knowledge, and apply their knowledge to the wiki-pages, to prove their accuracy by setting them according to the project standards, systematizing and referring to the reliable sources.

2. The level of information culture of the citizen plays an important role in the knowledge society. In the modern era of rapidly developing technology, the moral values, ideologies, beliefs and attitudes of others must be respected. Preserving these moral values and conveying them to future generations is an important issue. A knowledge society can be formed when people always want to acquire new knowledge and when the society has the ability to stimulate and satisfy their needs. The Wiki concept is capable to meet the needs of the people with innovative knowledge. Providing the links to a variety of non-existing web pages is one of the best ways to attract the people to the new knowledge-building process.

3. Provision of information, education and communication is an important aspect in organizing the knowledge society. The policy of innovation in scientific, technological and socio-economic fields, the ethical attitude to science and technology, the principles of adaptation to education in global information space are in line with the wiki concept.

4. The national interests (state interests) should be taken into consideration in the knowledge society. The process of collecting and transmitting knowledge requires the revision of old data from both technical and humanitarian sciences and the adoption of new fundamental methodological principles used in decision-making. Enhancing historical research in humanitarian fields by the scientists and the use of wiki technologies in various fields, such as governance, social management, education, communication, intercultural communication and integration, can contribute to a more effective solution of problems in these areas.

5. In the knowledge society, the protection of the state information space, against the backgrounds of interstate and global security, and the placement of national information resources in the virtual space are of particular importance. Thus, all means of social media, including popular wiki projects should be used in this process. 
Today, many companies use wiki-projects as a knowledge base that replaces the traditional statistical Internet sites, and these projects are more popular than ordinary websites. Collectively gathering the information on the wiki pages and grounding on impartiality and trustworthy sources enables the trust in the wiki-community within the society. The analysis of different opinions in the subject area indicates that the role of the wiki-community in the knowledge society, the behavior of individual social groups, which constitute the wiki-community or its leading force, is not entirely studied.

Activity of wiki-community is a significant factor in effecting the public consciousness and social processes in the society. Knowledge and skills of the society members, their planned activities, coordination and operational management based on defined principles and rules may also affect the involvement of the society as a social force in building the knowledge society and protecting the information security.

The power of the Wiki-community depends on its members' eagerness to work for wikiprojects and to participate in the meetings and discussions related to the problem solution. In wikicommunity, no one can force others to do anything. This community is structured to be easily used by the individual members and groups for their own purposes. Since the above-mentioned features of the wiki-society are the same as in the knowledge society, wiki-community can play a significant role in the formation of the knowledge society.

\section{Conclusion}

Research shows that wiki technology is a very successful project for building collective knowledge. Activities of wiki-community are in line with the basic principles and philosophy of the knowledge society. Thus, the main goal of the knowledge society concept is to create appropriate conditions for the joint use of knowledge, and to provide equal information access for all. This concept is fully consistent with the working principle of the wiki-projects. However, in the knowledge society, formed within the wiki-environment, only the knowledge supporting the ideology of the most active groups of this society is expanding. However, this is against the principles of the knowledge society. The solutions for this problem must be determined throughout the development of process of the knowledge society. .

Building the knowledge society on the Internet is closely linked to the social media and, first of all, to the wiki technologies. Building a healthy and active wiki-community is an important issue for expanding its role in the formation of the knowledge society. The activity of the wikicommunity with high cultural and spiritual values and knowledge can affect the formation of a knowledge society, the expansion of socio-economic relations, and the extension of the national information resources on the Internet and, finally, the rise of the political and economic influence of the state in the world.

\section{References}

1. Alguliyev R.M., Aliguliyev R.M., Alakbarova I.Ya. Wikimetric studies: current state and prospects // Telecommunications, 2014, No5, pp. 15-31.

2. Wagner C., Karen S. K. Cheung, Rachael K. F., Stefan B. Building Semantic Webs for e-government with Wiki technology // Journal of Electronic Government, vol. 3, issue 1, 2007, pp. 36-55.

3. Pekárek M., Pötzsch S. A comparison of privacy issues in collaborative workspaces and social networks // Journal of the Identity in the Information Society, 2009, vol. 2, issue 1, pp. 81-93.

4. Richter A., Koch M., Social software-Status quo und Zukunft // Technischer Bericht Nr., Fakultät für Informatik, Universität der Bundeswehr München, 2007, 48 p.

5. Cress U., Kimmerle J. A systemic and cognitive view on collaborative knowledge building with wikis // Computer-Supported Collaborative Learning. International Society of the Learning Sciences, Inc.; Springer Science \& Business Media, 2008, pp. 105-122. 
6. Alguliyev R.M., Aliguliyev R.M., Alakbarova I.Y. Extraction of hidden social networks from wiki-environment involved in information conflict // International Journal of Intelligent Systems and Applications (IJISA), 2016, vol. 8, no.2, pp. 20-27.

7. http://www.oed.com/view/Entry/267577

8. Leuf B., Cunningham W. The Wiki Way: Quick Collaboration on the Web. Laflin, PA: Addison-Wesley, 2001, $200 \mathrm{p}$.

9. Tapscott D., Williams D. A., Wikinomics: How Mass Collaboration Changes Everything. 2007, $324 \mathrm{p}$.

10.Peacock T., Fellows G., Eustace K., The quality and trust of wiki content in a learning community // School of Computing \& Mathematics, Charles Sturt University, 2007, pp. 822-832.

11. Moskaliuk J., Kimmerle J., Cress U. Collaborative knowledge building with wikis: The impact of redundancy and polarity // Journal of Computers \& Education, 2012, vol. 58, no. 4, pp. 1049-1057.

12. Lane R. The decline of politics and ideology in a knowledgeable society // American Sociological Review, 1966, vol. 31, no. 5, pp. 649-662

13. Drucker P.F. Post-Capitalist Society. N.Y., Harper-Collins Publishers, 1993, 232 p.

14. Delokarov K.Kh. Is the "knowledge-based society" a new type of society? Sat. Sci. Tr. RAS. INION, M., 2010, 234 p.

15. Drucker P.F. The New Society: The Anatomy of Industrial Order. N.Y., Transaction Publishers, 1993, $362 \mathrm{p}$.

16. Earl M.J. Knowledge Management Strategies: Toward a Taxonomy // Journal of Management Information Systems, 2001, vol.18, issue 1, pp.215-233.

17. Towards knowledge societies. UNESCO World Report. Paris: UNESCO Publishing House, 2005, 240 p.

18. http://www.az.wikipedia.org/wiki/Vikipediya: Beş əsas prinsip

19. http://www.wikimediafoundation.org/wiki/Home

20. Alakbarova I.Y. The basics of the Wikipedia virtual encyclopedia. Baku, "Information Technologies" publishing house, 2017, 205 p.

21. http://www.en.wikipedia.org/wiki/Wikipedia:Creating_a_bot 